\title{
A secondary data analysis of qualitative study of individualized treatment regimen for North Indians living with human immunodeficiency virus- 1
}

\section{Sushanta Kumar Barik}

National JALMA Institute for Leprosy \& Other Mycobacterial Diseases

Avi Kumar Bansal

National JALMA Institute for Leprosy \& Other Mycobacterial Diseases

Ashwini Yadav

National JALMA Institute for Leprosy \& Other Mycobacterial Diseases

Srikanth Prasad Tripathy

National Institute for Research in Tuberculsosi

Tej Pal Singh

Sarojini Naidu Medical College

\section{Srikanta Jena}

Ravenshaw University

\section{Shripad A Patil}

National JALMA Institute for Leprosy \& Other Mycobacterial Diseases

Keshar Kunja Mohanty ( $\sim$ keshar63@yahoo.com )

National JALMA Institute for Leprosy \& Other Mycobacterial Diseases

\section{Research Article}

Keywords: HIV, AIDS, Genotyping, treatment failure, NRTI, NNRTI

Posted Date: October 18th, 2021

DOl: https://doi.org/10.21203/rs.3.rs-744951/v2

License: (c) (1) This work is licensed under a Creative Commons Attribution 4.0 International License.

Read Full License 


\section{Abstract}

The follow-up of fifty-seven patients were conducted after a confirmation genotyping test. The secondary data analysis was done on the data available to observe the correlation between the CD4 counts and viral loads (One of the markers of clinical outcome) of the individual North Indian patients infected with HIV-1 Subtype $C$. The drug resistance mutations in individual patient were analysed through the drug resistance database, Stanford University, USA. The data of resistance associated with drugs, CD4 counts, viral loads of the individual patient was compiled and statistically analysed for drug resistance pattern profiles using Microsoft Excel 2016 and SPSS Version 22. The normality of data was checked by Shapiro-Wilk test $(p<0.05)$. The study starting and endpoint data on CD4 counts, viral loads and drug resistance patterns associated with multiple first-line ART was available for 24 north Indian patients. The starting and study endpoint data on CD4 counts and drug resistance patterns associated with multiple first- line ART was available for 33 North Indian patients. The study indicative that the recommendation of policy to provide a tailor-made individualized regimen to each patient under AIDS control program.

\section{Introduction}

Acquired immunodeficiency syndrome (AIDS) is a chronic syndrome caused by human immunodeficiency virus (HIV). The first-line antiretroviral therapy regimen was implemented by National AIDS Control Organization sponsored ART centre in India in the year $2004{ }^{1}$. It is comprised of two nucleoside reverse transcriptase inhibitors (NRTIs) and a non-nucleoside reverse transcriptase inhibitor (NNRTI) ${ }^{2,3}$. If the patients fail the first-line antiretroviral therapy (ART), they were put on second line ART like Atazanavir, Ritonavir, etc. The national program in India has supported the clinical and immunological monitoring of first-line ART patients by routine CD 4 count and viral load monitoring ${ }^{3}$. The 90-90-90 Strategy can be successful by implementing CD 4 counts, viral load and genotyping to assess human immunodeficiency virus drug resistance (HIVDR) in a national program ${ }^{4}$. The harrowing time spent by patients with drug resistance mutations without genotyping test can have a significant impact on the life of the patients ${ }^{5}$. Genotyping is the most common method of gene sequencing for the detection of HIV drug resistance mutations ${ }^{6}$. Commercial and in-house methods are widely used for the genotypic test of HIV-1 drug resistance ${ }^{7}$.

Genotypic drug resistance testing could be useful in patients who were initiated with first-line ART and identify PLHIV with virological failure. Based on genotypic drug resistance testing, appropriate regimen can be initiated during the period of treatment. WHO's report on nationally representative surveys on pretreatment HIV drug resistance (PDR) between 2014 and 2016 suggests greater than 10\% PDR to NNRTI was reported by 6 out of 11 countries. The estimated prevalence of NNRTI resistance among all patients on ART varied between $5 \%$ and $28 \%$. At present, antiretroviral treatment (ART) failure is monitored through CD4 counts, viral loads and clinical profile by National AIDS Control Programme in India. Currently, drug resistance testing by molecular methods is not routinely done in the initiation of treatment or monitoring 
of response to ART. The study suggests, the broader scale implementation research for the use of the tailor-made suitable drugs after an individualized genotyping test in national programs in India.

\section{Materials And Methods}

\section{Study design, treatment Policy and collection of samples}

At antiretroviral therapy centre, Sarojini Naidu Medical College, Agra, North India, 57 patients on the first line antiretroviral therapy between December 2009 to November 2016 were tested for drug resistance by genotypic study. Patients were followed up till August 2019. All patient's information was collected using the own designed patient information leaflet ${ }^{8,9}$. The selection of patients for genotyping was based on either of CD 4 counts ( $<350$ cubic/mm), WHO clinical staging of HIV/AIDS and viral load ( $\geq 1000$ copies $/ \mathrm{ml}$ ). This is a secondary analysis of de-identified data of 57 patients on CD4 counts, viral loads and drug resistance patterns. Data of all patients on HIV drug resistance, pre-treatment and interim CD4 count and viral load was not available. Out of 57 patients, 51 were found to be drug resistant. Data on pre-treatment and study endpoint CD4 count, viral load and HIV drug resistance were available for 24 patients while only starting CD4 counts with viral loads and study endpoint CD4 counts of 33 patients was available.

\section{Ethics approval and Informed consent}

The HIV drug resistance study was approved by the institutional ethics committee. The Institute ethics committee is representing as the guidelines of Indian Council of Medical Research ${ }^{9}$. The study was approved by the Institutional Review Board that is Institute Human Ethics Committee of National JALMA Institute for Leprosy and Other Mycobacterial Diseases, (Indian Council of Medical Research), Dr. M. Miyazaki Marg, TajGanj, Agra-282004. The registration number of institute Human Ethics Committee was ECR/257/Inst/ UP/ 2013. The ethics committee meeting was held on 27.5.2016 at National JALMA Institute for Leprosy and Other Mycobacterial Diseases, Agra for the project entitled "Characterization of drug resistant HIV-1 mutants of Agra region, India by genomic and proteomic approaches". Renewal of registration of Institute Human Ethics Committee of National JALMA Institute for leprosy and Other Mycobacterial Diseases was done by Central Drugs Standard Control Organization (CDSCO) with registration no. ECR/257/Inst/UP/2013/RR-20 dated $19^{\text {th }}$ June 2020 and provisional registration with department of Health Research, Ministry of Health and Family Welfare, Govt. of India, File numberEC/NEW/INST/2020/792 dated $27^{\text {th }}$ July 2020.

The study protocol and informed consent was approved by Institute Human Ethics Committee of National JALMA Institute for Leprosy and Other Mycobacterial Diseases, (Indian Council of Medical Research), Dr. M. Miyazaki Marg, Taj Ganj, Agra-282004. The informed consent of each study patient was taken by the information leaflet.

\section{Viral load and genotyping analysis}


Viral load was estimated using the Abbott automated m2000rt instrument. The genotyping was performed for first-line ART failure at the National Institute for Research in Tuberculosis, Chennai by using the WHO dried blood spot protocol 2010. The modified polymerase reaction conditions and primers of the WHO dried blood spot protocol 2010 was used in the genotyping study. The sequenced product of $935 \mathrm{bp}$ PR and 750bp RT gene fragment of HIV-1 was taken for genotyping. The drug resistance mutation of each sample was identified and interpreted using the Stanford HIV drug resistance database. (http://hivdb.stanford.edu/pages/algs/sierra _sequence.html) and was already reported ${ }^{10}$.

\section{Statistical analysis}

After analysis of the drug resistance mutations in individual patients, the data of individuals were compiled and analysed using Microsoft Excel 2016 and SPSS Version 22. The normality of data was checked by Shapiro-Wilk test $(p<0.05)$. It showed that CD 4 counts and viral loads are not normally distributed. The frequency analysis was done when resistance was found in more than one first-line antiretroviral drugs. The Wilcoxon signed rank test was used for hypothesis testing for CD4 counts and viral loads.

\section{Results}

All 57 HIV-1 infected North Indian patients were tested by genotyping and the drug resistance mutations were analysed in individual patients. Fifty-one North Indian patients were detected with drug resistance mutations (DRMs) associated with NRTIs and NNRTIs. The CD4 counts and viral loads were considered as prognostic markers to assess the treatment outcome. The north Indian patients received different regimens of the mentioned drugs as per their clinical profile and adverse drug events during the treatment duration. Data on pre-treatment and study endpoint CD4 counts, viral loads, and HIV drug resistance were available for 24 north Indian patients. All 24 north Indian patients showed resistance to one or more than one first-line anti-retro viral drugs [Table 1 (a)]. Out of 24 patients, three patients died during the treatment. One of them had decreased both CD4 counts and viral loads. The CD4 counts decreased and viral load increased for the rest of two patients. In the rest 21 patients, in the presence of drug resistance, both CD 4 counts and viral load declined in two patients while CD 4 count decreased and viral load increased in 7 patients. Both CD4 counts and viral load increased in 3 patients, whereas increased CD 4 counts and decreased viral load was observed in 12 patient's despites of the drug resistance. Out of 24 patients, 16 patients were shifted on a second-line regimen after the detection of first-line HIV drug resistance in them. AL/ATV/RTV had been started in one patient. But the patient died during the course of treatment. There was a decline in both CD 4 counts and viral loads. SL/ATV/RTV had been started in two patients, TL/ATV/RTV in 8 patients and ZL/ATV/RT in 5 patients. There was no significant difference between starting and endpoint CD4 count (' $p$ ' value 0.391 ) as well as viral load (' $p$ ' value 0.616 ).

Table 1 (a): Drug resistance pattern of patients 


\begin{tabular}{|c|c|c|c|c|c|c|c|c|c|c|c|c|}
\hline Sr. No. & Drugs & $\begin{array}{l}\text { First line ART failure } \\
\text { drugs }\end{array}$ & $\mid \begin{array}{l}\text { No. of } \\
\text { patients }\end{array}$ & $\left|\begin{array}{l}\text { CD4 } \\
\text { count at } \\
\text { DSA }\end{array}\right|$ & $\begin{array}{l}\text { End } \\
\text { point } \\
\text { CD4 } \\
\text { count }\end{array}$ & $\begin{array}{l}\text { Viral } \\
\text { load at } \\
\text { DSA }\end{array}$ & \begin{tabular}{|l} 
End \\
point \\
viral \\
load
\end{tabular} & $\begin{array}{l}\text { Second line } \\
\text { regimen }\end{array}$ & \begin{tabular}{|l|} 
Total \\
treattment \\
duration \\
(Months)
\end{tabular} & $\begin{array}{l}\text { Adherence } \\
\text { (\%) }\end{array}$ & $\begin{array}{l}\text { Status of } \\
\text { the } \\
\text { patie nt }\end{array}$ & Remark \\
\hline 1 & $\begin{array}{l}\text { Abacavir, Lamivudine } \\
\text { Nevirapine, Tenofovir } \\
\text { Efavirenz }\end{array}$ & $\begin{array}{l}\text { Abacavir, Lamivudine } \\
\text { Nevirapine, Tenofovir } \\
\text { Efavirenz }\end{array}$ & 1 & 134 & 20 & 371277 & 16842 & AL/ATV/RTV & 26.6 & 96.27 & Dead & $\begin{array}{l}\text { CD4 decrease } \\
\text { Viral load de crease }\end{array}$ \\
\hline 2 & $\begin{array}{l}\text { Stavudine, Lamivudine } \\
\text { Nevirapine, Zidovudine } \\
\text { Tenofovir, Efavirenz }\end{array}$ & \begin{tabular}{|l|} 
Stavudine, Lamivudine, \\
Nevirapine,Tenofovir, \\
Efavirenz
\end{tabular} & 1 & 271 & 218 & 5427 & 40426 & No & 48.28 & 99.31 & Alive & $\begin{array}{l}\text { CD4 decrease } \\
\text { Viral load increase }\end{array}$ \\
\hline 3 & $\begin{array}{l}\text { Stavud ine, Lamivudine, } \\
\text { Nevirapine, Tenofovir, } \\
\text { Efavirenz }\end{array}$ & $\begin{array}{l}\text { Lamivudine,Nevirapine, } \\
\text { Efavirenz }\end{array}$ & 1 & 64 & 522 & 7964 & TND & ZL/ATV/RTV & 80.29 & 9871 & Alive & $\begin{array}{l}\text { CD4 increase } \\
\text { Viral load decrease }\end{array}$ \\
\hline 4 & $\begin{array}{l}\text { Stavud ine, Lamivudine, } \\
\text { Nevirapine,Tenofovir, } \\
\text { Efavirenz }\end{array}$ & $\begin{array}{l}\text { Lamivudine, Tenofovir, } \\
\text { Efavirenz, Nevirapine }\end{array}$ & 1 & 53 & 100 & 1900 & 24391 & No & 53.1 & 987 & Alive & $\begin{array}{l}\text { CD4 increase } \\
\text { Viral load increase }\end{array}$ \\
\hline 5 & $\begin{array}{l}\text { Stavud ine, Lamivudine, } \\
\text { Nevirapine,Zidovudine, } \\
\text { Efavirenz }\end{array}$ & Nevirapine,Efavirenz & 1 & 110 & 268 & 36177 & $<20$ & No & 62.26 & 99.5 & Alive & $\begin{array}{l}\text { CD4 increase } \\
\text { Viral load de crease }\end{array}$ \\
\hline \multirow[t]{3}{*}{6} & \multirow[t]{3}{*}{$\begin{array}{l}\text { Teno fovir, Lamivudine, } \\
\text { Efavirenz }\end{array}$} & \begin{tabular}{|l|} 
Lamivudine,Nevirapine, \\
Efavirenz
\end{tabular} & 1 & 200 & 218 & 22864 & 336119 & ZL/ATV/RTV & 15.19 & 94.4 & Alive & \begin{tabular}{|l} 
CD4 increase \\
Viral load increase
\end{tabular} \\
\hline & & \multirow{2}{*}{ Efavirenz } & 1 & 83 & 279 & 373820 & 43 & SL/ATV/RTV & 16 & 95.17 & Alive & $\begin{array}{l}\text { CD4 increase } \\
\text { Viral load decrease }\end{array}$ \\
\hline & & & 1 & 121 & 198 & 89339 & 12891 & No & 1811 & 95.63 & Alive & $\begin{array}{l}\text { CD4 Increase } \\
\text { Viral load decrease }\end{array}$ \\
\hline \multirow[t]{3}{*}{7} & \multirow[t]{3}{*}{$\begin{array}{l}\text { Teno fovir, Lamivudine, } \\
\text { Efavirenz, Nevirapine }\end{array}$} & $\begin{array}{l}\text { Lamivudine,Nevirapine, } \\
\text { Efavirenz }\end{array}$ & 1 & 141 & 78 & 6353 & 670719 & ZL/ATV/RTV & 4219 & 97.86 & Dead & $\begin{array}{l}\text { CD4 Decrease } \\
\text { Viral load increase }\end{array}$ \\
\hline & & \multirow{2}{*}{ Nevirapine,Efavirenz } & 1 & 103 & 148 & 519326 & 537350 & ZL/ATV/RTV & 39.23 & 98.8 & Alive & \begin{tabular}{|l|} 
CD4 increase \\
Viral load increase
\end{tabular} \\
\hline & & & 1 & 133 & 192 & 2066 & 1189 & ZL/ATV/RTV & 29.13 & 98.45 & Alive & \begin{tabular}{|l|} 
CD4 increase \\
Viral load decrease
\end{tabular} \\
\hline 8 & $\begin{array}{l}\text { Teno fovir, Lamivudine, } \\
\text { Nevirapine,Zido vudine, } \\
\text { Efavirenz }\end{array}$ & $\begin{array}{l}\text { Zudovu dine, Lamivudie, } \\
\text { Nevirapine,Efavirenz }\end{array}$ & 1 & 143 & 192 & 465426 & 2256 & TL/ATV/RTV & 41.5 & 98.38 & Alive & \begin{tabular}{|l|} 
CD4 increase \\
Viral load decrease
\end{tabular} \\
\hline 9 & $\begin{array}{l}\text { Zdovudine, } \\
\text { Lamivudine, } \\
\text { Efavirenz,Stavudine, Nev } \\
\text { irapine,Tenofovir }\end{array}$ & $\begin{array}{l}\text { Zidovu dine, Lamivudine, } \\
\text { Teno fovir,Stavudine, } \\
\text { Efavirenz, Nevirapine }\end{array}$ & 1 & 83 & 49 & 9983 & 11847 & No & 72.2 & 99.14 & Dead & \begin{tabular}{|l|} 
CD4 decrease \\
Viral load increase
\end{tabular} \\
\hline 10 & $\begin{array}{l}\text { Zidovudine, } \\
\text { Lamivudine, } \\
\text { Efavirenz, Tenof ovir }\end{array}$ & $\begin{array}{l}\text { Zdovudine, } \\
\text { Lamivudine, } \\
\text { Efavirenz, Tenof ovir } \\
\end{array}$ & 1 & 295 & 82 & 2950 & $<20$ & SL/ATV/RTV & 41.5 & 93.59 & Alive & \begin{tabular}{|l|} 
CD4 decrease \\
Viral load decrease
\end{tabular} \\
\hline \multirow[t]{2}{*}{11} & \multirow[t]{2}{*}{$\begin{array}{l}\text { Żdovudine, } \\
\text { Lamivudine, } \\
\text { Efavirenz, Nevirapine }\end{array}$} & \begin{tabular}{|l|} 
Zidovudine, \\
Lamivudine, \\
Efavirenz, Nevirapine \\
\end{tabular} & 1 & 204 & 223 & 62572 & 60 & TL/ATV/RTV & 57.7 & 98.19 & Alive & $\begin{array}{l}\text { CD4 increase } \\
\text { Viral load decrease }\end{array}$ \\
\hline & & $\begin{array}{l}\text { Zdovudine, } \\
\text { Lamivudine, } \\
\text { Efavirenz, Nevirapine } \\
\end{array}$ & 1 & 76 & 222 & 6266 & TND & TL/ATV/RTV & $\pi .11$ & 98.32 & Alive & $\begin{array}{l}\text { CD4 increase } \\
\text { Viral load decrease }\end{array}$ \\
\hline \multirow[t]{4}{*}{12} & \multirow[t]{4}{*}{$\begin{array}{l}\text { Zudovudine, } \\
\text { Lamivudine, Nevirapine }\end{array}$} & Zdovu dine, Lamivudine & 1 & 137 & 90 & 44125 & 286866 & TL/ATV/RTV & 24.1 & 97.34 & Alive & $\begin{array}{l}\text { CD4 decrease Viral } \\
\text { load increase }\end{array}$ \\
\hline & & Nevirapine & 1 & 290 & 160 & 10913 & 472121 & TL/ATV/RTV & 31.15 & 99.39 & Alive & \begin{tabular}{|l|} 
CD4 decrease \\
Viral load increase
\end{tabular} \\
\hline & & \begin{tabular}{|l|} 
Zudovudine, \\
Lamivudine, Nevirapine
\end{tabular} & 1 & 112 & 161 & 50603 & 48887 & TL/ATV/RTV & 35.6 & 95.81 & Alive & $\begin{array}{l}\text { CD4 increase; Viral } \\
\text { load decrease }\end{array}$ \\
\hline & & Zdovudine, Nevir apine & 1 & 253 & 174 & 9008 & 248242 & TL/ATV/RTV & 53.9 & 95.95 & Alive & $\begin{array}{l}\text { CD4 decrease } \\
\text { Viral load increase }\end{array}$ \\
\hline 13 & $\begin{array}{l}\text { Zidovu dine, Lamividine, } \\
\text { Nevirapine,Stavudine, } \\
\text { Teno fovir, Efavirenz } \\
\end{array}$ & $\begin{array}{l}\text { Zdovudine, Lamivudine, } \\
\text { Stavud ine, Nevir apine }\end{array}$ & 1 & 240 & 436 & 30694 & TND & No & 61.13 & 99.7 & Alive & \begin{tabular}{|l|} 
CD4 increase \\
Viral load decrease
\end{tabular} \\
\hline \multirow[t]{2}{*}{14} & \multirow{2}{*}{$\begin{array}{l}\text { Żdovudine, } \\
\text { Lamivudine, } \\
\text { Nevirapine,Tenofovir, } \\
\text { Efavirenz }\end{array}$} & \multirow{2}{*}{$\begin{array}{l}\text { Zidovudine, } \\
\text { Lamivudine, } \\
\text { Nevirapine,Tenofovir, } \\
\text { Efavirenz }\end{array}$} & 1 & 312 & 193 & 12344 & 56094 & No & 47.1 & 9811 & Alive & $\begin{array}{l}\text { CD4 decrease } \\
\text { Viral load increase }\end{array}$ \\
\hline & & & 1 & 267 & 357 & 11847 & TND & No & 66.2 & 99.3 & Alive: & $\begin{array}{l}\text { CD4 increase } \\
\text { Viral load decrease }\end{array}$ \\
\hline 15 & $\begin{array}{l}\text { Zudovudine, } \\
\text { Lamivudine, Nevirapine, } \\
\text { Efavirenz }\end{array}$ & $\begin{array}{l}\text { Lamivudine, } \\
\text { Nevirapine, Efavirenz }\end{array}$ & 1 & 265 & 272 & 151379 & 118265 & TL/ATV/RTV & 40.21 & 97.14 & Alive & $\begin{array}{l}\text { CD4 increase } \\
\text { Viral load decrease }\end{array}$ \\
\hline
\end{tabular}

Table 1 (b) Drug resistance pattern of patients whose end point viral load was not available 


\begin{tabular}{|c|c|c|c|c|c|c|c|c|c|c|}
\hline $\begin{array}{l}\text { Sr. } \\
\text { No. }\end{array}$ & Drugk Regimen & Drug resistance & $\begin{array}{c}\text { No. of } \\
\text { patients }\end{array}$ & \begin{tabular}{|c|} 
CD4 \\
count at \\
DSA
\end{tabular} & \begin{tabular}{|c|} 
End point \\
CD4 \\
count
\end{tabular} & $\begin{array}{c}\text { Viral load } \\
\text { at DSA }\end{array}$ & \begin{tabular}{|l|}
$\begin{array}{c}\text { Second } \\
\text { Line } \\
\text { Regimen }\end{array}$ \\
\end{tabular} & \begin{tabular}{|c|} 
Ad he re nce \\
$(\%)$
\end{tabular} & $\begin{array}{c}\text { Status of } \\
\text { the patient }\end{array}$ & Peemark \\
\hline 1 & $\begin{array}{c}\text { SLavudie, Lamivudine, } \\
\text { Elavirene, Nevirapine, } \\
\text { Zidovudine } \\
\end{array}$ & $\begin{array}{l}\text { Lamivudine, Efavirenz, } \\
\text { Nevirapine, Zidovedine }\end{array}$ & 1 & 129 & 143 & 17032 & No & 96.52 & $\begin{array}{l}\text { Lost to } \\
\text { follow up }\end{array}$ & $\begin{array}{c}\text { CD4 } \\
\text { Increased }\end{array}$ \\
\hline 2 & $\begin{array}{c}\text { Stavudine, Lamivudine, } \\
\text { Efaviren2, Nevirap ine, } \\
\text { Tenofovir }\end{array}$ & Nev vir apine, Elavir ens & 1 & 58 & 98 & 373829 & No & 98.68 & Dee and & $\begin{array}{c}\mathrm{CD4} \\
\text { Increased d }\end{array}$ \\
\hline 3 & \begin{tabular}{|l|} 
Stavudine, Lamivud ine, \\
EfaviremL, Zdeovudine, \\
Nev vir appine, Ternofovir \\
\end{tabular} & $\begin{array}{c}\text { Stavudine, Lamivudine, } \\
\text { Eravirene, Zidovudine, } \\
\text { Nevirapine }\end{array}$ & 1 & 87 & 175 & 34015 & No & 96.99 & Dee and & $\begin{array}{c}\text { CD4 } \\
\text { Increased }\end{array}$ \\
\hline 4 & $\begin{array}{c}\text { SLavudine, Lamivudines, } \\
\text { Efaviren2, Zidovudine, } \\
\text { Nevirapine }\end{array}$ & Stavudine, Zidovudine & 1 & 258 & 79 & 206019 & $\begin{array}{c}\text { TL/ATV/ } \\
\text { RTV }\end{array}$ & 90.45 & De and & $\begin{array}{c}\text { CD4 } \\
\text { Dencres aned }\end{array}$ \\
\hline 5 & 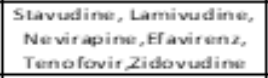 & Zidovudine, Lamivudiner & 1 & 134 & 295 & 4300 & No & 99.63 & Aliver & $\begin{array}{c}\text { CD4 } \\
\text { Increased d }\end{array}$ \\
\hline 6 & \begin{tabular}{|c|}
$\begin{array}{c}\text { Stavudine, Lamivudine, } \\
\text { Nevirap ine, } \\
\text { Zidovudine, Tenof ovir, } \\
\text { Eravirenz }\end{array}$ \\
\end{tabular} & $\begin{array}{l}\text { Zidovudine, Tenofovir, } \\
\text { Stavudine, Lamivudine, } \\
\text { Nevirapine, Efavirene }\end{array}$ & 1 & 203 & 15 & 315008 & No & 98.47 & Aliver & $\begin{array}{c}\text { CD4 } \\
\text { Dencres anet d }\end{array}$ \\
\hline \multirow{6}{*}{7} & \multirow{6}{*}{$\begin{array}{c}\text { Tenofovir, Lamivudine, } \\
\text { Efaviremz }\end{array}$} & Lamivudine, EFavirenz & 1 & 265 & 140 & 140398 & No & 88 & Dee and & C.D4 \\
\hline & & \multirow{2}{*}{$\begin{array}{l}\text { Lamivudine, Tenofovir, } \\
\text { Efavirene }\end{array}$} & 1 & 435 & 133 & 162109 & No & 86.73 & Alive & \begin{tabular}{|c|} 
CD4 \\
Dencreaked \\
\end{tabular} \\
\hline & & & 1 & 348 & 442 & 85743 & No & 96.68 & Aliver & \begin{tabular}{|c|} 
CD4 \\
increasedd \\
\end{tabular} \\
\hline & & \multirow{3}{*}{ NONE } & 1 & 3088 & 246 & 51301 & No & 86.99 & Alive & \begin{tabular}{|c|} 
CD4 \\
Decrerenesd \\
\end{tabular} \\
\hline & & & 1 & 385 & 300 & 175904 & No & 87.68 & De and & \begin{tabular}{|c|} 
CD4 \\
Descresined \\
\end{tabular} \\
\hline & & & 1 & 27 & 40 & 2095 & No & 89.7 & De iad & \begin{tabular}{|c|} 
CDA \\
increased \\
\end{tabular} \\
\hline \multirow{9}{*}{8} & \multirow{9}{*}{$\begin{array}{l}\text { Tenofovit, Lamivudine, } \\
\text { Elavirene, Nevir apine }\end{array}$} & $\begin{array}{l}\text { Ternofovir, Lamivudine, } \\
\text { Eravirenz, Nevirapines }\end{array}$ & 1 & 194 & 143 & 54937 & No & 90.12 & Aliver & $\begin{array}{c}\text { CD4 } \\
\text { Durcrenated } \\
\end{array}$ \\
\hline & & $\begin{array}{c}\begin{array}{c}\text { Zidovudine, Lamivudine, } \\
\text { Terno fovir, Nevir apine, } \\
\text { Efavireme }\end{array} \\
\end{array}$ & 1 & 37 & 91 & 253388 & No & 98.32 & Alive & $\begin{array}{c}\text { CD4 } \\
\text { increased }\end{array}$ \\
\hline & & Tenofovir & 1 & 640 & 142 & 10586887 & $\begin{array}{c}\text { SL/ATV/R } \\
\text { TV } \\
\end{array}$ & 89.66 & Alive & $\begin{array}{c}\text { CD4 } \\
\text { Dencreaned } \\
\end{array}$ \\
\hline & & NONE & 1 & 168 & 234 & 103158 & \begin{tabular}{|c|} 
ZL/ATV/R \\
TV \\
\end{tabular} & 93.27 & Alive & \begin{tabular}{|c|} 
C.D4 \\
increased d \\
\end{tabular} \\
\hline & & $\begin{array}{c}\text { Lamivud ine, Nevir apine, } \\
\text { Elavirenc }\end{array}$ & 1 & 264 & 37 & 203217 & No & 95.27 & Dee and & \begin{tabular}{|c|} 
CD4 \\
Decirenawd \\
\end{tabular} \\
\hline & & $\begin{array}{l}\text { Lamivudine, Te no fovir, } \\
\text { Eravirenz, Nevir apine }\end{array}$ & 1 & 168 & 188 & 163223 & No & 92.22 & Alive & $\begin{array}{c}\text { CD4 } \\
\text { ingreased } \\
\end{array}$ \\
\hline & & Nervirapine, Efavirent & 1 & $\nexists 2$ & 58 & 310718 & $\mathrm{No}$ & 91.59 & Den and & \\
\hline & & $\begin{array}{c}\text { lamivudine, Tenofovir, } \\
\text { Efavirene }\end{array}$ & 1 & 231 & 254 & 1063 & No & 93.81 & Alive & $\begin{array}{c}\text { CD4 } \\
\text { increased d }\end{array}$ \\
\hline & & $\begin{array}{c}\text { Zidovudine, Lamivudine, } \\
\text { Nevirapine, Efavirenz }\end{array}$ & 1 & 55 & 199 & 28007 & No & 90.32 & Aliver & $\begin{array}{c}\text { CD4 } \\
\text { Increaseed } \\
\end{array}$ \\
\hline \multirow{2}{*}{9} & \multirow{2}{*}{$\begin{array}{l}\text { Zidovudine, Lamivudine, } \\
\text { Eravirene, Stavudine, } \\
\text { Nevir ap ine, Terno fovit }\end{array}$} & $\begin{array}{l}\text { Zidovudine, Lamivudine, } \\
\text { Efavirene, Stavudine, } \\
\text { Nevinapine, Tenofovir }\end{array}$ & 1 & 27 & 64 & 697164 & No & 90.24 & Aliver & $\begin{array}{c}\text { CD4 } \\
\text { Increased }\end{array}$ \\
\hline & & $\begin{array}{c}\text { Zdovudine, Tenofovit, } \\
\text { Stavudine, Elavirene, } \\
\text { Nevirapine }\end{array}$ & 1 & 103 & 161 & 2200 & No & 95.61 & Alive & $\begin{array}{c}\text { CD4 } \\
\text { Increaserd }\end{array}$ \\
\hline 10 & \begin{tabular}{|} 
Zidovudine, Lamivudines, \\
Elavirene, Nevirapine
\end{tabular} & $\begin{array}{l}\text { Lamivudine, EFavirenz, } \\
\text { Nevirapine }\end{array}$ & 1 & $\mathbf{B}$ & 958 & 200151 & No & 98.89 & Aliver & $\begin{array}{c}\text { CD4 } \\
\text { increased } \\
\end{array}$ \\
\hline \multirow{2}{*}{11} & \multirow{2}{*}{$\begin{array}{c}\text { Zidovedine, Lamivudines, } \\
\text { Efaviren2, Nevirapine, } \\
\text { Tenofovir }\end{array}$} & NONE & 1 & 172 & 219 & 1566552 & $\begin{array}{c}\text { TL/ATV/ } \\
\text { RTV }\end{array}$ & 97.39 & Aliver & $\begin{array}{c}\text { CD4 } \\
\text { Increased d }\end{array}$ \\
\hline & & Zidovudine, Lamivudiner & 1 & 222 & 510 & 159004 & No & 82.21 & Aliver & \begin{tabular}{|c|} 
CD4 \\
Increased d \\
\end{tabular} \\
\hline 12 & \begin{tabular}{|c|} 
Zidovudine, Lamivudine, \\
Nevirapine, Stavudines, \\
Tenofovir, Elavirenz \\
\end{tabular} & $\begin{array}{c}\text { Zidovudine, Lamivudine, } \\
\text { Nervirapine, Stavudine, } \\
\text { Tenofovir, Efavirenz }\end{array}$ & 1 & 207 & 306 & 1997 & No & 97.23 & Aliver & $\begin{array}{c}\text { CD4 } \\
\text { increased }\end{array}$ \\
\hline \multirow{2}{*}{13} & \multirow{2}{*}{$\begin{array}{c}\text { Zidovudine, Larnivudine, } \\
\text { Nevirapine, Teno fovit, } \\
\text { Efaviremz }\end{array}$} & $\begin{array}{l}\text { Zidovudine, Ter nofovir, } \\
\text { Nevir apine, Elavirenz }\end{array}$ & 1 & 253 & 314 & 1195 & No & 95.56 & Alive & $\begin{array}{c}\text { CD4 } \\
\text { Increased d } \\
\end{array}$ \\
\hline & & $\begin{array}{l}\text { Lamivudine, Teno fovir, } \\
\text { Efavirenz, Nevir apine }\end{array}$ & 1 & 150 & 266 & 1694 & No & 98.51 & Aliver & \begin{tabular}{|c|} 
CD4 \\
Increased d \\
\end{tabular} \\
\hline 14 & $\begin{array}{l}\text { Zidovudine, Lamivudines, } \\
\text { Nevir ap ine, Ter no fovir }\end{array}$ & $\begin{array}{c}\text { Zidovudine, Lamivodine, } \\
\text { Nevirapine, Tenofovir, } \\
\text { Efavirene }\end{array}$ & 1 & 87 & 142 & 3698 & No & 99.25 & Alive & $\begin{array}{c}\mathrm{CD4} \\
\text { increased d }\end{array}$ \\
\hline \multirow{2}{*}{15} & \multirow{2}{*}{$\begin{array}{c}\text { Zidovudine, Lamivudine, } \\
\text { Nevirapine, Tenofovir, } \\
\text { Efavirenz }\end{array}$} & NONE & 1 & 157 & 380 & 2901 & $\begin{array}{c}\text { PA.L/LPV/ } \\
\text { FTV }\end{array}$ & 97.97 & Alive & \begin{tabular}{|c|} 
CD4 \\
increaserd \\
\end{tabular} \\
\hline & & $\begin{array}{c}\text { Zidovudine, Nevirapine, } \\
\text { Efavirenc }\end{array}$ & 1 & 255 & 362 & 1219 & No & 95.41 & Alive & \begin{tabular}{|c|} 
CD4 \\
increaserd
\end{tabular} \\
\hline 16 & $\begin{array}{c}\text { Zidovudine, Laminudine, } \\
\text { Nevirapine, Elavirenz, } \\
\text { Tenofovir }\end{array}$ & $\begin{array}{l}\text { Lamivudine, Efavirenz, } \\
\text { Nevinapines }\end{array}$ & 1 & 54 & 191 & 2950 & No & 97.09 & De and & $\begin{array}{c}\text { CD4 } \\
\text { Increased d }\end{array}$ \\
\hline
\end{tabular}

Data of viral load at study end point was not available for 33 patients [Table 1 (b)]. It shows that although CD4 counts improved in 24 patients, 5 of them died. Two patients on TLE regimen who died during treatment did not show resistance to any ART drug. 18 patients who were alive had improved CD4 count (11 patients had less than $300 / \mathrm{mm}^{3}$ and 7 patients had it $\geq 300 / \mathrm{mm}^{3}$ ). Five patients were shifted on 
second-line regimen out of which one died during the treatment. There was no significant difference between starting and endpoint median CD4 count (' $p$ ' value 0.189 ) of these 33 North Indian patients.

Median duration between diagnosis and initiation of treatment was 8 weeks (Range: 1 to 460 weeks). Median duration of treatment was 41.5 months (range 10.3 to 86.6 months) and treatment adherence was $97.34 \%$ (range $82.21 \%$ to $99.7 \%$ ).

Table 2: Drug resistance frequency analysis (multiple observations due to different regimens)

\begin{tabular}{|c|c|c|c|c|c|}
\hline \multirow{2}{*}{$\begin{array}{l}\text { Sr. } \\
\text { No. }\end{array}$} & \multirow{2}{*}{$\begin{array}{c}\text { Class of ART } \\
\text { drug }\end{array}$} & \multirow{2}{*}{$\begin{array}{l}\text { Name of the } \\
\text { drug }\end{array}$} & $\begin{array}{c}\text { Endpoint viral load } \\
\text { available }\end{array}$ & $\begin{array}{c}\text { Endpoint viral load not } \\
\text { available }\end{array}$ & \multirow[t]{2}{*}{ Total } \\
\hline & & & $\begin{array}{l}\text { Number out of } 24 \\
\text { (\%) }\end{array}$ & Number out of 33 (\%) & \\
\hline 1 & \multirow[t]{2}{*}{ NNRTI } & Nevirapine & $18(75)$ & $19(57.57)$ & 37 \\
\hline 2 & & Efavirenz & $16(66.67)$ & $22(66.66)$ & 38 \\
\hline 3 & \multirow[t]{5}{*}{ NRTI } & Lamivudine & $16(66.67)$ & $19(57.57)$ & 35 \\
\hline 4 & & Zidovudine & $10(41.67)$ & $13(39.39)$ & 23 \\
\hline 5 & & Tenofovir & $5(20.83)$ & $13(39.39)$ & 18 \\
\hline 6 & & Stavudine & $3(12.5)$ & $6(18.18)$ & 9 \\
\hline 7 & & Abacavir & $1(4.16)$ & $0(0.0)$ & 1 \\
\hline
\end{tabular}

When the drug resistance patterns of these patients were studied, Efavirenz and Nevirapine resistance was observed in most of these patients followed by Lamivudine and Zidovudine (Table 2). Few patients were resistant to tenofovir, stavudine and abacavir. Thus, resistance to NNRTIs was observed more frequently than NRTIs.

\section{Discussion}

Treatment failure can be assessed by an immunological, virological or clinical measure. The establishment of the measurement of RNA copies is a surrogate marker for HIV disease progression ${ }^{11}$. The low-level RNA copies may not predict virologic failure, however, the high RNA copy number is a risk of treatment failure ${ }^{12}$. The successful antiretroviral treatment is specifically defined as viral suppression in 
people living with HIV (PLHIV) ${ }^{13}$. The viral load has become the cornerstone for evaluating the success of anti-retroviral therapy in clinical and research. Few North Indian patients in the present study had higher endpoint viral loads than baseline in presence of resistance to the first line ART drugs.

It has been suggested that, CD 4 count of individual patients after therapy confirms, the risk of the progression of AIDS or death ${ }^{14}$. But in present study, decline in CD 4 count was observed irrespective of drug resistance status of North Indian patients. Few North Indian patients died during treatment although their CD4 count was increased.

The clinical goal for each patient requires an effective, active, tolerable and minimal side effects regimen to achieve maximal clinical benefits to avoid the definition of treatment failure. In this study, drug resistance was found to various first-line drugs such as zidovudine, lamivudine, tenofovir, stavudine, efavirenz and nevirapine. HIV drug resistance has been monitored by affordable HIV drug resistance testing in Sub-Saharan Africa ${ }^{15}$. From the multi-centric study in India, it was concluded that Nevirapine therapy is reasonable alternative for efavirenz ${ }^{16}$. In this North Indian population study, resistance to Nevirapine and Efavirenz was most commonly observed.

Tenofovir is an important regimen included in the first-line antiretroviral therapy. Tenofovir based virological monitoring predicted treatment failure for patients ${ }^{17}$. In this study, 18 North Indian patients were found to be resistant to Tenofovir ${ }^{18}$, addressed the monitoring of the degree of genotypic resistance to zidovudine. In this study, Lamivudine resistance was found in 35 North Indian patients. Zidovudine resistance was found in 23 North Indian patients. Similar finding was observed in a study where in zidovudine and lamivudine combinatorial therapy, both zidovudine and lamivudine therapy failure were marked in HIV-1 infected North Indian patients ${ }^{19}$.

Stavudine exhibits antiretroviral activity as a monotherapy or combination therapy to manage the adults with HIV infection ${ }^{20}$. The rate of treatment failure for stavudine/lamivudine /nevirapine was determined in Asian region ${ }^{21}$. In this study, out of 11 North Indian patients on SLE/SLN combination therapy, 9 North Indian patients were developed resistance to stavudine.

Abacavir is a suitable antiretroviral drug with lamivudine for treatment of HIV patients ${ }^{22}$.One patient was treated with ALN regimen and Abacavir treatment failure was observed in that one patient.

The management of HIV resistance differs in lower middle-income countries compared to high income countries. It has been recommended that HIV drug resistance testing should like a point of care test ${ }^{23}$. This study utilized WHO dried blood spot protocol 2010 polymerase chain reaction and sequencing primers. Thermal conditions are useful in genotyping test which can reduce the cost of the test.

In India, the antiretroviral therapy is still introduced at an advanced stage of disease without drug resistance testing. This might be the main cause of mortality of HIV patients. The studies assessing the relationship between DRMs and their effect on treatment outcome are limited. An insight into HIV drug 
resistance pattern and its effect on prognosis of AIDS in individual patients is important. It can also enable the health care providers to monitor HIV drug resistance over the period of treatment. However, utility of such test at the level of national health program needs to be studied in detail before formulation of policy.

\section{Conclusion}

This is a secondary data analysis based on available records of a very few North Indian patients. The effect of drug resistance on CD4 count, viral suppression, and clinical outcome could not be established. To support evidence-based policy decision, determinants of HIV drug resistance, and its effect on ART outcome need to be explored further in a structured manner in North Indian population. The introduction of molecular drug resistance testing in the program in the presence of an appropriate evidence may lead to proper management of healthcare resources for HIVDR management in North Indian population. The implementation science research on a larger scale may assist in prioritizing interventions for HIV drug resistance control. Monotherapy or combination therapy based on individualized genotyping test in North Indian population may prove an effective intervention if planned on the basis of evidence generated through such research.

\section{Declarations}

\section{Consent for publication}

Not applicable

\section{Availability of Data and Materials}

Not required

\section{Competing interests}

All authors declared no conflict of interest.

\section{Funding}

Indian Council of Medical Research, Govt. of India has funded the senior research fellowship to Mr. Sushanta Kumar Barik and contingencies grant. The sanction file number of the project: 80/990/2015ECD-I.

\section{Author's contributions}

SKB: Manuscript write up and data collection, data analysis, AKB: Concept design for secondary data analysis, AY: Statistical Analysis of this paper and manuscript write up, SPT: Method design of the study, 
TPS: Patient study, SJ: Review the paper, SAP: Review the paper, KKM: Review the paper, concept design and manuscript write up.

Acknowledgements: We thanks to the staff members of ICMR-NIRT, Chennai for their assistance in the viral load and genotyping study in HIV-1 patient's sample.

\section{References}

1. National guidelines for implementation of antiretroviral therapy, New Delhi:NACO; 2004:1-151.

2. Ministry of Health and Family welfare of India NACO. Guidelines for Prevention and Management of Common Opportunistic Infections/ Malignancies among HIV-Infected Adult and Adolescent.2007185 .

3. Ministry of Health and Family welfare of India NACO. Antiretroviral Therapy Guidelines for HIVInfected Adults and Adolescents. 2013:40-45.

4. Unaids 90-90-90: an ambitious treatment target to help end the AIDS epidemic. UNAIDS JC2684. Joint United Nations Programme on HIV/AIDS 2014 Feb 16.

5. Van Vaerenbergh, K. Study of the impact of HIV genotypic drug resistance testing on therapy efficacy. Verh K Acad Geneeskd Belg, 63, 447-473 (2001).

6. Shafer, R. W. Genotypic testing for human immunodeficiency virus type 1 drug resistance. Clin. Microbiol. Rev, 15, 247-277 (2002).

7. Chaturbhuj, D. N., Nirmalkar, A. P., Paranjape, R. S. \& Tripathy, S. P. Evaluation of a cost-effective inhouse method for HIV-1 drug resistance genotyping using plasma samples. PLoS One, 9, 1-8 (2014).

8. Barik, S. K. et al. Practical Challenges in Implementing a Simplified Leaflet for HIV Patients in Resource Poor Settings: The Practice towards Public Health. J. Gen. Pract, 06, 1-3 (2018).

9. Ethical guidelines for biomedical research on human participants. Indian Council of Medical Research. (2006)1-120.

10. Barik, S. K. et al. Detection of Drug Resistance Mutations in the Reverse Transcriptase Gene of HIV-1Infected North Indian Population Failing First-Line Antiretroviral Therapy "A Follow-Up Cohort Study". AIDS Res. Hum. Retroviruses, 00, 1-10 (2021).

11. Haubrich, R. H. et al. A randomized study of the utility of human immunodefidency virus RNA measurement for the management of antiretroviral therapy. Clin. Infect. Dis, 33, 1060-1068 (2001).

12. Kiweewa, F. et al. HIV virologic failure and its predictors among HIV-infected adults on antiretroviral therapy in the African Cohort Study. PLoS One, 14, 1-16 (2019).

13. Karade, S. K. et al. Antiretroviral resistance following immunological monitoring in a resource-limited setting of western India: A cross-sectional study. PLoS One, 12, 1-11 (2017).

14. Young Cd4 cell count and the risk of AIDS or death in HIV-infected adults on combination antiretroviral therapy with a suppressed viral load: A longitudinal cohort study from COHERE.PLOS Med.9, (2012). 
15. Inzaule, S. C. et al. Affordable HIV drug-resistance testing for monitoring of antiretroviral therapy in sub-Saharan Africa. Lancet Infect. Dis, 16, e267-e275 (2016).

16. Sinha, S. et al. Nevirapine- versus Efavirenz-based antiretroviral therapy regimens in antiretroviralnaive patients with HIV and Tuberculosis infections in India: A multi-centre study. BMC Infect. Dis, 17, 1-8 (2017).

17. Shet, A. et al. Virological efficacy with first-line antiretroviral treatment in India: predictors of viral failure and evidence of viral resuppression. Trop. Med. Int. Heal, 20, 1462-1472 (2015).

18. Zazzi, M. et al. Zidovudine resistance mutations and human immunodeficiency virus type 1 DNA burden: Longitudinal evaluation of six patients under treatment., 24, 419-425 (1996).

19. Miller, V. et al. Dual resistance to zidovudine and lamivudine in patients treated with zidovudinelamivudine combination therapy: Association with therapy failure. J. Infect. Dis, 177, 1521-1532 (1998).

20. Moyle, G. J. \& Stavudine Pharmacology, clinical use and future role. Expert Opin. Investig. Drugs, 6, 191-200 (1997).

21. Zhou, J. et al. Experience with the use of a first-line regimen of stavudine, lamivudine and nevirapine in patients in the TREAT Asia HIV observational database. HIV Med, 8, 8-16 (2007).

22. Achenbach, C. J., Scarsi, K. K. \& Murphy, R. L. Abacavir/lamivudine fixed-dose combination antiretroviral therapy for the treatment of HIV. Adv. Ther, 27, 1-16 (2010).

23. Noguera-Julian, M. HIV drug resistance testing - The quest for Point-of-Care. EBioMedicine, 50, 1112 (2019). 